\title{
A Physical Model for DT-MRI Based Connectivity Map Computation ${ }^{\star}$
}

\author{
Erdem Yörük ${ }^{1}$, Burak Acar ${ }^{1}$, and Roland Bammer ${ }^{2}$ \\ 1 Department of Electrical\&Electronics Engineering, Boğaziçi University, Turkey \\ erdem. yoruk, acarbu@boun.edu.tr \\ 2 LUCAS MRS/I Center, Department of Radiology, Stanford University, CA, USA
}

\begin{abstract}
In this study we address the problem of extracting a robust connectivity metric for brain white matter. We defined the connectivity problem as an energy minimization task, by associating the DT-field to a physical system composed of nodes and springs, with their constants defined as a function of local structure. Using a variational approach we formulated a fast and stable map evolution, which utilizes an anisotropic kernel smoothing scheme equivalent to a diffusion PDE. The proposed method provides connectivity maps that correlate with normal anatomy on real patient data.
\end{abstract}

\section{Introduction}

Magnetic Resonance Diffusion Tensor Imaging (DT-MRI) is the only known technique that provides information about the geometry of fibrous structures, such as the fibers in the human brain, in vivo. DT-MRI is based on measuring the diffusion of water molecules. The diffusion process is approximated by a second order tensor. It was shown that at fiber positions, the principal eigenvector of this tensor is correlated with the underlying fibrous structures' orientation [3]. So, DT-MRI has been accepted as a promising tool to get information about the connections in human brain. Despite its promise, DTI has its drawbacks: Low order approximation to the diffusion process and insufficient spatial resolution.

There are two basic approaches in utilizing the information DTI data provides: Fiber tractography and connectivity mapping. The former attempts to answer the following question: Can we reconstruct the fiber that passes through a given seed point? Among different approaches, the Runge-Kutta method has been widely accepted [1]. However, fiber tracking is prone to cumulative errors and can not overcome the partial volume effect $[2,3]$. The second group of approaches attempts to utilize the nature of the DTI data, i.e. the diffusion process, by estimating a connectivity map. They consider each and every possible connection with weights set by the DTI data. Koch et al. used a Monte-Carlo simulation of the random walk model [4], yet their approach uses successive jumps whose curvature is restricted to be less than 90 degrees and requires multiple runs for

\footnotetext{
* This work was in part supported by EU 6th Framework SIMILAR NoE (www.similar.cc), TÜBİTAK KARIYER-DRESS Project (104E035) and Boğaziçi University Research Funds (SK/DTIcad).
} 
generating the final connectivity map. Hagmann et al. extended this method in a supervised fashion by incorporating the white matter fiber tract curvature [5], again treating the problem as a random process. Chung et al. applied adaptive kernel smoothing of the transition probabilities of the underlying diffusion process, which is governed by the DTI tensor field [6]. Their approach relatively being faster and more deterministic than the first two, is shown to be equivalent to the Monte Carlo method. It remains to be a numerical diffusion simulation, and needs to be masked with the FA map in order to be a heuristic approximation of connectivity. Batchelor et al. solved the anisotropic diffusion equation set by the DTI data with the initial conditions being a set of seed points set to an initial nonzero (unity) concentration of virtual diffusing particles [7]. Lenglet et al., on the other hand, recasted the connectivity problem to Riemannian differential geometry framework where they defined their local metric tensor using the DTI data and solved for geodesics [8].

In this work, we propose aphysical setup of unit-mass nodes (voxels) and springs. The constants of spring linking adjacent nodes are set according to the local connectedness of the node pair. The local connectedness is measured using the so called mutual diffusion coefficent. It approximates the ellipsoidal volume overlap of a given tensor pair in $\Re^{3}$. The system is perturbed by setting the seed node to constant potential and computing the stationary potential map, which is the connectivity map, evoked. The method is evaluated on real petient data.

\section{Method}

In general, connectivity can be interpreted as a measure which is proportional to the qualitative similarity and spatial proximity of the units contained in data to be analyzed. In the case of DT images, where we are trying to reveal a functional connectivity, qualitative assessment of tensors becomes crucial in order to construct an appropriate model. We have to consider some certain features embedded in a DT image as indicators of connectivity. In general, the connectivity measure between two tensors can be visualized in terms of the volume overlap of their ellipsoids centered at the corresponding voxel coordinates. This interpretation will take the relative features of tensors into consideration such as their respective locations, sizes, orientations and shapes. A similar but simpler metric, that we proposed and used in this study is the so-called distance scaled mutual diffusion coefficient $K$. Given two tensors $\mathbf{D}_{1}$ and $\mathbf{D}_{2}$, located at $\mathbf{r}_{1}$ and $\mathbf{r}_{2}$, respectively, we define their connectivity $K_{12}$ as:

$$
K_{12}=\frac{\left[\left(\mathbf{v}^{T} \mathbf{D}_{1} \mathbf{v}\right)\left(\mathbf{v}^{T} \mathbf{D}_{2} \mathbf{v}\right)\right]^{\gamma}}{\delta^{2}}
$$

where $\mathbf{v}=\left(\mathbf{r}_{1}-\mathbf{r}_{2}\right) / \delta$ and $\delta=\left\|\mathbf{r}_{1}-\mathbf{r}_{2}\right\|_{2}$. Thus, $K$ reflects the mutual influence of tensor pairs by giving the distance scaled product of their diffusion coefficients evaluated in the unit direction of their Euclidean link and raised to the power $\gamma$, which will be used as a tuning parameter. For the time being we take $\gamma$ to be 1 . We can construct a physical system, based on $K_{i j}$ 's which reflect the connectivity pattern within DTI data. 


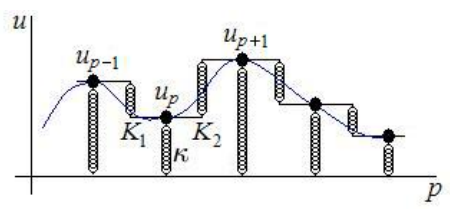

Fig. 1. Illustration of proposed spring system for a 1D curve (solid line), dots represent the nodes, spring constants are given for the specific node $u_{p}$

Let $\Omega=[0, a] \times[0, b] \times[0, c] \subseteq \Re^{3}$ be our image domain, and let $\mathbf{D}: \Omega \rightarrow$ $\Re^{3} \times \Re^{3}$ be the given tensor field. We propose that the sought connectivity map $u: \Omega \rightarrow \Re$ with respect to a given seed point $\left(x_{0}, y_{0}, z_{0}\right) \in \Omega$ is the stationary pattern of a physical spring system defined as follows: Each voxel of the domain corresponds to a node and potential at seed node $u\left(x_{0}, y_{0}, z_{0}\right)$ is set to 1 , which is kept constant in time. For each pair of adjacent nodes, a spring is associated with a stiffness, that is set to the mutual diffusion coefficient (Eqn. 1) evaluated for the adjacency of that particular pair. Thus, if we consider 6neighborhood $\left(N_{6}\right)$ on a regular $3 \mathrm{D}$ grid with $\delta$-spacing in all directions, this model will correspond to 6 "neighbor springs" acting on each node and a node at $\mathbf{r}_{i}=(x, y, z)^{T}$ would share the following spring constant with respect to its immediate neighbor $\mathbf{r}_{j}=(x-\delta, y, z)^{T}$ :

$$
K_{1}(x, y, z)=\frac{\left(\mathbf{e}_{1}^{T} \mathbf{D}(x, y, z) \mathbf{e}_{1}\right)\left(\mathbf{e}_{1}^{T} \mathbf{D}(x-\delta, y, z) \mathbf{e}_{1}\right)}{\delta^{2}}
$$

where $\mathbf{e}_{1}=(1,0,0)^{T} / /\left(\mathbf{r}_{i}-\mathbf{r}_{j}\right)$ is the unit vector of the orthonormal basis. Remaining constants of neighbor springs $K_{2}, \ldots, K_{6}$ are defined similarly with $\mathbf{e}_{2}=(0,1,0)^{T}$ and $\mathbf{e}_{3}=(0,0,1)^{T}$. We set spring elongations as the local directional differences of $u$ (for instance as $u(x, y, z)-u(x-\delta, y, z)$ for $K_{1}$ ), such that they force $u$ to be equal to the corresponding immediate neighbor. Finally, an extra spring, the so-called "ground spring" with a constant stiffness $\kappa$, is also attached to all nodes, with the elongation being $u$ and forcing $u$ to be 0 . This is necessary to justify our model for revealing connectivity, which will be explained next. The resulting spring system can be visualized in Figure 1 for a 1D curve. With this model, total potential energy stored will be given as:

$$
V_{\text {springs }}=\frac{1}{2} \kappa u^{2}+\sum_{n=1}^{6} V_{n}
$$

where $V_{1}=\frac{1}{2} K_{1}[u(x, y, z)-u(x-\delta, y, z)]^{2}$ and $V_{2}, \ldots V_{6}$ are defined similarly. In order to associate this potential energy to the point $(x, y, z)$ rather than to the springs, the latter six energy terms due to the neighbor connections should further be halved, as if they are equally shared with the corresponding neighbor. We let $\delta \rightarrow 0$ assuming that we have infinite resolution, such that $V$ can be approximated to a continuous function, enabling us to interpret the system better. Inserting $K_{n}$ 's common denominator $\delta^{2}$ and taking the limit, we obtain: 


$$
V\left(u, u_{x}, u_{y}, u_{z}\right)=\frac{1}{2}\left(\kappa u^{2}+d_{11}^{2} u_{x}^{2}+d_{22}^{2} u_{y}^{2}+d_{33}^{2} u_{z}^{2}\right)
$$

where $d_{j j}(j=1,2,3)$ are the diagonal elements of $\mathbf{D}$ as can easily be derived from Equation 2. We can put the current problem into a variational one, where the connectivity map $u$ with respect to the seed at $\left(x_{0}, y_{0}, z_{0}\right)$ can be found by minimizing the following energy functional:

$$
J(u)=\int_{\Omega} V\left(u, u_{x}, u_{y}, u_{z}\right) d \Omega
$$

assuming Neumann boundary conditions on $\partial \Omega$, and an extra seed condition $u\left(x_{0}, y_{0}, z_{0}\right)=1$. Corresponding Euler-Lagrange equation is:

$$
\kappa u-\frac{d}{d x}\left(d_{11}^{2} u_{x}\right)-\frac{d}{d y}\left(d_{22}^{2} u_{y}\right)-\frac{d}{d z}\left(d_{33}^{2} u_{z}\right)=0
$$

It is easy to show that fixing $u\left(x_{0}, y_{0}, z_{0}\right)$ to 1 for an interior point of $\Omega$, does not change the Euler-Lagrange equation since $J(u)$ can be rewritten as a sum of several integrals evaluated at Cartesian subregions, each having $\left(x_{0}, y_{0}, z_{0}\right)$ at their boundaries and sharing the same integrand. The physical interpretation of our model is as follows: A connected spring system, which is only lifted at $\left(x_{0}, y_{0}, z_{0}\right)$ to a constant level of 1 and kept there, achieves its stationary pattern by minimizing its total potential energy. Nodes other than the seed try to come to zero due to the ground springs, but they will be lifted up as well, in proportion to their connectivity to the stationary seed node. Hence, the stationary pattern will be equivalent to the sought connectivity map with respect to the seed node.

Temporally evolving a given initial $u_{0}$ at a rate determined by the negative of the left term of Equation 6, by a steepest descent scheme, we obtain the following PDE:

$$
u_{t}=-\kappa u+\frac{d}{d x}\left(d_{11}^{2} u_{x}\right)+\frac{d}{d y}\left(d_{22}^{2} u_{y}\right)+\frac{d}{d z}\left(d_{33}^{2} u_{z}\right)=-\kappa u+\nabla \bullet(\widetilde{\mathbf{D}} \nabla u)
$$

where $\widetilde{\mathbf{D}}$ is a diagonal matrix with squared diagonal entries of $\mathbf{D}$. The same equation could be obtained with another $\widetilde{\mathbf{D}}$, using different neighborhood (and/or spring) definitions. In any case, resulting PDE can be considered as a modified diffusion process, with a seed condition $u\left(x_{0}, y_{0}, z_{0}\right)=1$ and an extra term $-\kappa u$, keeping $u$ attached to the ground. Thus, as $t \rightarrow \infty, u$ does not get totally flat. If we interpret the model in $2 \mathrm{D}$, a structure sensitive tent-like pattern will be obtained with its maximum at the seed.

Numerical implementation of such a map evolution scheme can easily be accomplished by anisotropic diffusion filters with the specified modifications and an initial map:

$$
u_{0}(x, y, z)=\left\{\begin{array}{cc}
1 & \text { if }(x, y, z)=\left(x_{0}, y_{0}, z_{0}\right) \\
0 & \text { otherwise }
\end{array}\right.
$$


Keeping the model in its discrete form from the beginning, we can get the discrete version of Equation 5 as:

$$
J(u)=\sum_{p}\left\{\kappa u_{p}^{2}+\frac{1}{2} \sum_{n=1}^{N} K_{p n}\left(u_{p}-u_{p n}\right)^{2}\right\}
$$

where $p$ is the voxel index and $N$ is the number of neighbors. $u_{p n}$ and $K_{p n}$ stand for the $n^{t h}$ neighbor of the $p^{t h}$ node and its associated spring constant, respectively. $\frac{1}{2}$ scales the potential energy of neighbor springs, since they are counted twice in the outer summation. Now, writing the discrete Euler-Lagrange equation of this functional, we obtain:

$$
\kappa u_{p}+\sum_{n}^{N} K_{p n}\left(u_{p}-u_{p n}\right)=0
$$

This equation says that along an extremal, the total spring force applied to each node is zero. This could also be observed by rewriting Equation 6 in a discrete form where space derivatives are replaced with central differences.

Seeking for the connectivity as a PDE evolution with diffusion filters, will bring the common trade-off between stability and rate of convergence. We propose, another map evolution technique, which mimics the diffusion process in the light of the balanced force condition imposed by discrete Euler-Lagrange equation. The idea is, not to calculate the update $u_{t}$, but iteratively solve for a new $u_{p}$, which makes $u_{t}$ vanish at $p$. "Balance in the neighborhood" condition dictated by Equation 10, locally and explicitly gives:

$$
u_{p}=\frac{\sum_{n}^{N} K_{p n} u_{p n}}{\kappa+\sum_{n}^{N} K_{p n}}
$$

This scheme with $\kappa=0$ would be the same as assigning a weighted neighborhood average to each $u_{p}$, but with a spatially varying kernel coming from the local image structure. In fact, this adaptive directional smoothing constitutes the essence of the diffusion filtering, hence it can be interpreted to be equivalent to the diffusion process [6]. Equation 11 has a similar computational cost to Equation 7, but a higher convergence rate. Moreover, stability is naturally provided by the bounds specified by local neighborhood of $u_{p n}$ 's.

\section{Results}

In order to validate the computed connectivity maps, we used a simple tracking module, that follows coherence directions in converged $u$. As in [9], we first compute the structure tensor of $u$, which is obtained by taking the tensor product of its gradient and componentwise smoothing the resulting matrix:

$$
\mathbf{S}(u)=G_{\sigma} *(\nabla u \otimes \nabla u)=\left[\begin{array}{lll}
\omega_{1} & \omega_{2} & \omega_{3}
\end{array}\right]\left[\begin{array}{ccc}
\lambda_{1} & 0 & 0 \\
0 & \lambda_{2} & 0 \\
0 & 0 & \lambda_{3}
\end{array}\right]\left[\begin{array}{c}
\omega_{1}^{T} \\
\omega_{2}^{T} \\
\omega_{3}^{T}
\end{array}\right]
$$


where $G_{\sigma}$ is a Gaussian kernel with variance $\sigma^{2} . \mathbf{S}(u)$ is a symmetric positive semidefinite matrix, which is invariant under sign changes of the gradient. Its magnitude ordered eigenvalues $\lambda_{1} \geq \lambda_{2} \geq \lambda_{3}$ give the amount of $u$ 's fluctuation, along the corresponding eigenvectors. Thus, as $\omega_{1}$ will be parallel to the smoothed gradient, the smallest eigenvector $\omega_{3}$, which is also called the coherent direction, will correspond to the orientation with least decrease in the connectivity. Starting from the seed, we can easily follow $\omega_{3}$ vectors for tracking the candidate fiber.

We used a real patient data in our experiments. The scans were single-shot EPI scans with diffusion encoding along 12 non-collinear directions plus one reference without diffusion-weighting. The FOV was $25-26 \mathrm{~cm}$, TE was minimum with partial k-space acquisition. TR was $\sim 10 \mathrm{~s}$ and b-value was $\sim 850 \mathrm{~s} / \mathrm{mm}^{2}$.

Experiments are comparatively conducted based on 26 neighborhood for different seed points. Note that, with $N_{26}$, spring constants for corners of a $3 \times 3 \times 3$

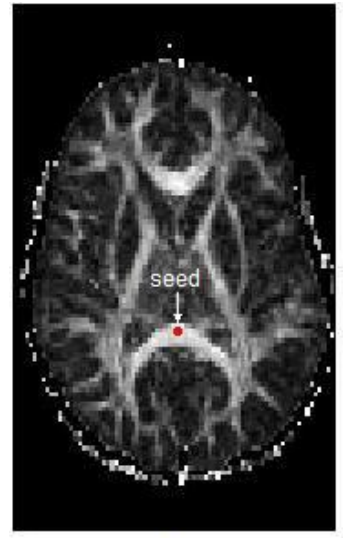

a

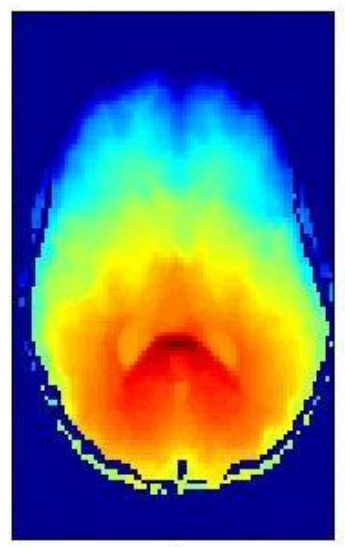

d

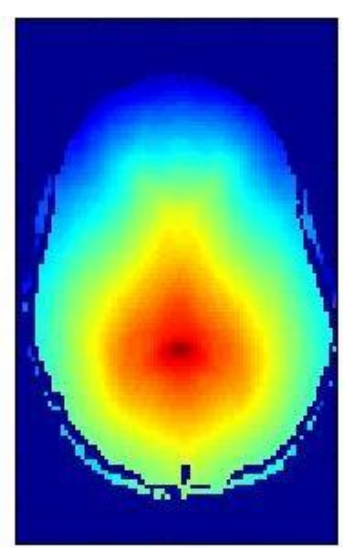

b

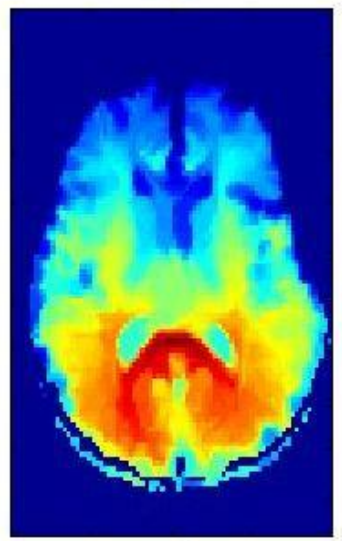

e

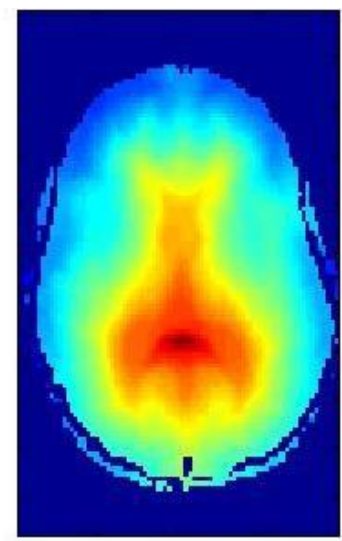

C

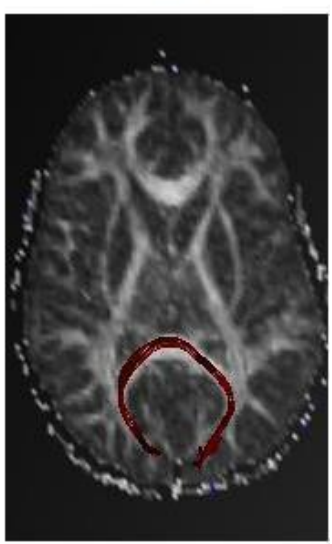

f

Fig. 2. a) Axial slice of FA map with the seed indicated, b) Converged connectivity map $(\gamma=1)$, c-e) Converged maps for $\gamma=2,4,10, \mathrm{f})$ computed tracts for $\gamma=10$ 
neighborhood cube, appear with weights $\frac{1}{2}$ and $\frac{1}{3}$ due to the denominator $\delta^{2}$. Stiffness for the ground springs is empirically taken to be $\frac{1}{100}$ of the mean neighbor stiffness of the whole domain and converged maps are visualized in the logscale. Convergence of the iterations was assumed when the mean absolute change in $u$ dropped below $10^{-4}$ with $u \in[0,1]$. Qualitative evaluation of the results were done based on the computed connectivity maps and the tracts computed from $u$ maps.

Figure 2 shows axial cross-sections of the computed 3D connectivity maps with different $\gamma$ values, ranging from 2 to 10 and the computed tracts. Note the correlation between the connectivity map and the brain anatomy as well as the assigned connectivity values with respect to the seed point. The anatomy is enhanced as the $\gamma$ increases. The tracts in Figure $2 \mathrm{f}$ were computed based on the coherence directions of $u$ as defined in [9]. They also correlate with the underlying fiber structure.

The overall computation time is independent of the number of seed points. It took less than 100 seconds with Matlab R14 using a PC with P4 $2.4 \mathrm{GHz}$ processor and $2 \mathrm{~GB}$ RAM to process a $128 \times 128 \times 38$ volume.

\section{Discussion}

In this study we proposed a novel method for computing the connectivity in white matter. We associated the DT-image to a physical spring system, and interpreted the underlying connectivity to be its stationary pattern. We followed a variational approach, which aims to minimize the total spring potential energy and showed that the PDE evolution scheme coming from the Euler-Lagrange equation corresponds to a modified diffusion process. Reformulating the energy minimization problem in the discrete domain enabled us to replace the PDE with an alternative directional averaging method, which guarantees stability and exhibits a higher convergence rate.

The proposed method is computationally inexpensive and provides several advantages. The model is valid for different definitions of neighborhood and spring constants. It provides a tuning parameter $\gamma$ that allows one to continuously trade-off between using the complete DTI data with using the principal diffusion direction only. At one end, our method corresponds to the tractography approach (large $\gamma$ ) and at the other hand it corresponds to complete connectivity model $(\gamma=1)$.

The proposed method allows to set multiple seed points, even regions. Thus, one can easily incorporate a-priori information about the anatomy into the connectivity map computation. Potentially, one can search for the most probable path between two given points by simply setting them as the seed points, computing the associated connectivity map and running a tractography algorithm on this map.

As mentioned above ground springs are necessary for preventing total flattening of $u$, such that we obtain meaningful maps that monotonically decrease as we get away from the seed. The stiffness $\kappa$ of the ground springs does not 
make much difference as long as it is nonzero. $\kappa$ should be sufficiently small, such that the farthest point of the brain can also attain a nonzero connectivity value and should be large enough so that the converged map does not become nearly

flat. We empirically set it to be $\frac{1}{100}$ of the mean of neighbor spring constants.

Further research is required to evaluate the method.

\section{References}

1. Tench, C.R., Morgan, P.S., Wilson, M., Blumhardt, L.D.: White Matter Mapping Using Diffusion Tensor MRI. Magn. Res. Med. 47 (2002) 967-972

2. Alexander, A.L., Hasan, H.M., Lazar, M., Tsuruda, J.S., Parker, D.L.: Analysis of Partial Volume Effects in Diffusion-Tensor MRI. Magn. Res. Med. 45 (2001) 770-780.

3. Basser, P.J., Pajevic, S., Pierpaoli, C., Duda, J., Aldroubi, A.: In vivo tractography using DT-MRI data. Magn. Res. Med. 44 ( 2000) 625-632

4. Koch, M.A., Norris, D.G., and Hund-Georgiadis, M.: An Investigation of Functional and Anatomical Connectivity using Magnetic Resonance Imaging. NeuroImage 16 (2002) 241-250.

5. Hagmann, P., Thiran, J.-P., Vandergheynst, P., Clarke, S., Meuli, R.: Statistical Fiber Tracking on DT-MRI Data as a Potential Tool for Morphological Brain Studies. ISMRM Workshop on Diffusion MRI: Biophysical Issues (2000).

6. Chung, M.K., Lazar, M., Alexander, A.L., Lu, Y., Davidson, R.: Probabilistic Connectivity Measure in Diffusion Tensor Imaging via Anisotropic Kernel Smoothing. University of Wisconsin, Dept. of Statistics (2003) Technical report no: 1081

7. Batchelor, P.G., Hill, D.L.G., Atkinson, D., Calamante, F., Atkinson, D.: Study of Connectivity in the Brain using the Full Diffusion Tensor from MRI. IPMI 2001, Lecture Notes in Computer Science 2082 (2001) 121-133.

8. Lenglet, C., Deriche, R., Faugeras, O.: Diffusion Tensor Magnetic Resonance Imaging: Brain Connectivity Mapping. INRIA (2003) Technical report no: 4983

9. Weickert, J.: Coherence-Enhancing Diffusion Filtering. International Journal of Computer Vision 31(2/3) (1999) 111-127 